\title{
An organic junction between the vein and membrane of the dragonfly wing
}

\author{
CHEN YingLong, WANG XiShu*, REN HuaiHui \& LI XuDong \\ Department of Engineering Mechanics, AML, School of Aerospace, Tsinghua University, Beijing 100084, China
}

Received January 5, 2011; accepted March 15, 2011

\begin{abstract}
A dragonfly wing consists of membranes and both longitudinal and cross veins. We observed the microstructure cross-section at several locations in the dragonfly wing using environmental scanning electron microscopy (ESEM). The organic nature of the junction between the vein and the membrane was clearly identifiable. The membrane was divided into two layers, the upper epidermis and the lower epidermis. These layers extend around the sandwich structure vein, and combine with the adjacent membrane at a symmetrical location along the vein. Thus, we defined this as an organic junction between the vein and the membranes. The organic junction is able to form a tight corrugation angle, which dramatically increases both the warping rigidity and the strength of the wing, but not the torsional rigidity. The torsional deformation is primarily controlled by the microstructure of the longitudinal veins, and is based on the relative rotation angle between the epidermal layer and the inner layer of the vein that forms the zigzag section.
\end{abstract}

dragonfly wing, vein, membrane, microstructure, organic junction

Citation: Chen Y L, Wang X S, Ren H H, et al. An organic junction between the vein and membrane of the dragonfly wing. Chinese Sci Bull, 2011, 56: 1658-1660, doi: 10.1007/s11434-011-4491-2

The dragonfly wing has long fascinated physicists and biologists. Both functional and optimized mechanical models have been constructed by scholars such as Wootton, Ellington, and Newman [1-3]. The studies to date have used mechanical models and fluid dynamics to study the aerodynamic performance and flight behaviors of the insect wing, forewing, or hindwing. However, the complex motions of flapping insects have not yet been adequately described. Similarly, no one has accurately measured the force imposed on the wing surface of flapping wings. Ennos [4] first highlighted the importance of torsion in the design of insect wings. This is because the inertia of the wing can cause it to rotate at the point of stroke reversal, resulting in the necessary changes in pitch among the longitudinal veins.

Recently, a number of studies have evaluated the microstructure and mechanical properties of the dragonfly wing vein and membrane. Wang et al. [5] first reported that the

*Corresponding author (email: xshwang @tsinghua.edu.cn) microstructure of the longitudinal vein is a complex sandwich consisting of chitinous shells and a protein layer with some fibrils. This allows the vein to endure greater bending loading and torsional deformation. Song et al. [6] concluded that the membrane is divided into three layers, a dorsal surface, a middle layer, and a ventral surface. These divisions were evident at the nano scale, along the orientation of the thickness of the membrane, and arose from their disparate functions. The thickness of these layers was $513.63 \pm 69.02$ $\mathrm{nm}, 1.93 \pm 0.18 \mu \mathrm{m}$, and $356.33 \pm 42.50 \mathrm{~nm}$, respectively.

The surface morphologies of moth and butterfly wings are closely related to their physical and chemical properties [7-10]. Zhao et al. [11] recently reported the morphologies of spikes and ripple wave of the longitudinal vein at both micro and nano scales. However, the structure of the joint between the vein and the membrane remains unclear. We investigated the structure of the connection between the vein and the membrane using pictures obtained by ESEM. Based on our observations, we discuss the self-adaptability 
of the organic junction and the effect of the microstructure on flight behaviors.

A dragonfly wing is generally considered to be composed of membranes and veins that are distinguished as cross veins and longitudinal veins (Figure 1). The longitudinal spars in the wings perform a similar function to manmade girders, that is, they resist the bending moment and torque in the wing when flapping. The corrugated structure of the wing was first studied by Hertel [12] who concluded that it was caused by the three dimensional (3-D) arrangement of the longitudinal veins. A cell is defined as the membranous region between the longitudinal and cross veins. Cross veins that link adjacent longitudinal veins are thinner, so their function tends to prevent the membrane between the spars from folding up [11]. To some extent, these veins can be viewed as support for structural corrugation [4].

We used Pantala flavesens that were captured on the campus of Tsinghua University. All individuals were maintained alive prior to the experiments. The ESEM (FEI Quanta 200) images ( $\times 2000$ magnification) of the wing's cross-section (Figure 2) reveal that the membrane is divided into two layers in the region outside the joint between the vein and the membrane. These layers are referred to as the upper epidermis and the lower epidermis. These layers differ from the three layers proposed by Song et al. (mentioned above) because of the differences in scale (nano versus micro). The dorsal surface is incorporated in the upper epidermis and the lower epidermis incorporates the ventral surface. However, for the latter, separating the ventral surface layer from the lower epidermis or the dorsal surface from the lower epidermis appears futile. Both the upper and lower epidermises extend around the sandwich structure vein and combine with the adjacent membrane at a symmetrical position along the vein. This point of connection is termed the organic junction between the membrane and the vein, and is very similar to the structure of a joint. The left and right membranes are distributed symmetrically along the vein (Figures 2 and 3). The left membrane grows across the sandwich vein, and then combines with the right membrane. Therefore, the membrane of the wing can be characterized as a continuous organic sheet, similar to the skin of an airfoil (Figure 4). This arrangement allows the formation

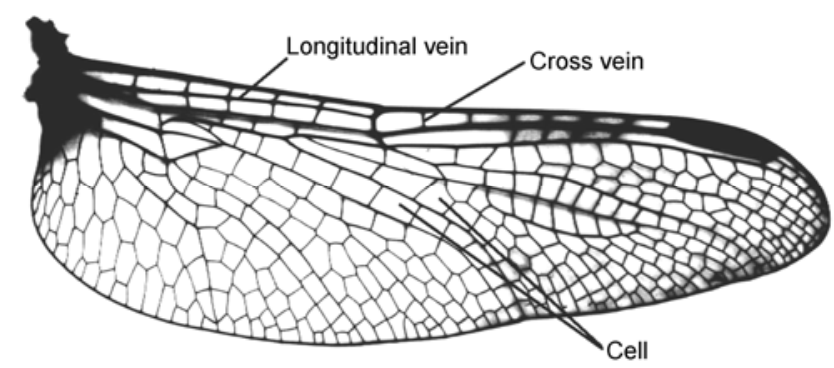

Figure 1 The skeleton of Pantala flavesens hindwing.
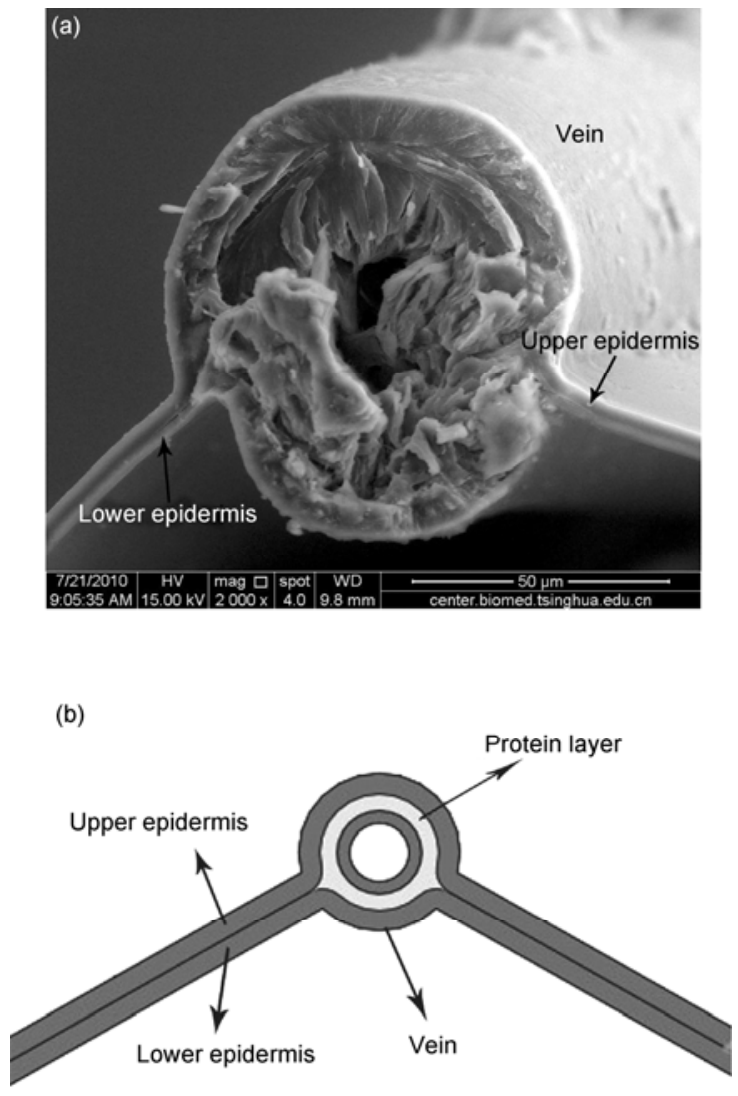

Figure 2 Microstructure of the wing's cross-section (a) and a model of the organic junction (b).

of a smooth effective profile for the wing. The thickness of both the upper epidermis and the lower epidermis (Figure 3(b)) was $3 \mu \mathrm{m}$. The thickness of the membrane and the diameter of the vein were $6 \mu \mathrm{m}$ and $55 \mu \mathrm{m}$, respectively.

The organic junction or self-adaptable joint is able to form ultra small corrugation angle (Figure 4), which dramatically increases both the warping rigidity and strength of the wing, but not the torsional rigidity [13]. However, the structure of the vein likely aids in resisting torque as the relative rotation angle between the epidermis layer and the protein layer of the vein is fixed.

The average value of parameter $\theta$ (the corrugation angle) can be as small as $50^{\circ}$ [14]. The ratio of the warping rigidity between an uncorrugated and corrugated wing is approximated as [6]

$$
\frac{\Gamma}{\Gamma_{R}}=(9 / 8)(c / t)\left(\cos ^{2} \theta / \sin \theta^{3}\right),\left(c / t>10, \theta \neq 90^{\circ}\right),
$$

where $\Gamma$ and $\Gamma_{R}$ represent the warping rigidity for an uncorrugated and corrugated wing, respectively, $c$ is the chord length, and $t$ is the wing thickness. This equation indicates that the ratio is a decreasing function of $\theta$. Thus, smaller $\theta$ values equate to increased warping rigidity. Fortunately, the organic joint between the membrane and vein was able to 

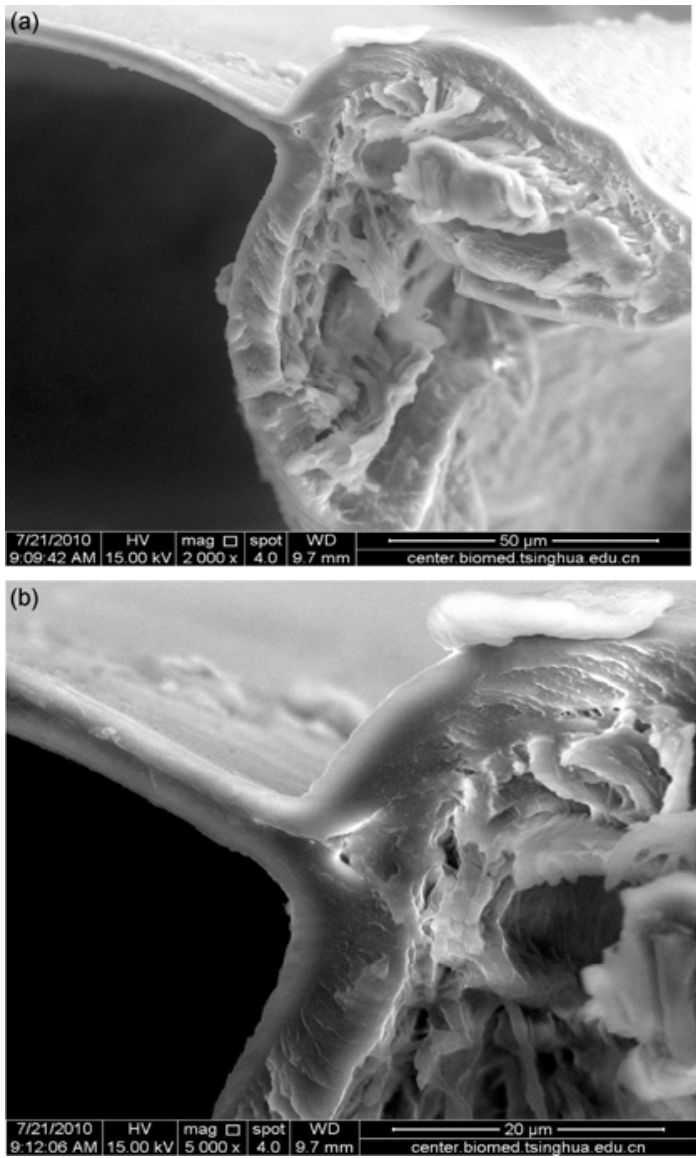

Figure 3 (a) Microstructure of the wing's cross-section at the leading edge; (b) a partial enlargement of (a) (×5000 magnification).

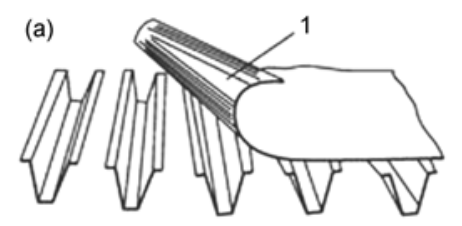

(b)

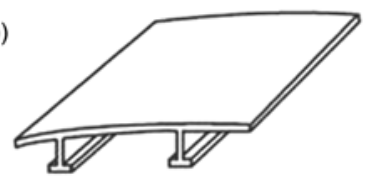

Figure 4 The airfoil skin. The label "1" represents the skin covering the surface which forms a smooth profile, as shown in (b).

afford the corrugation.

In summary, there are reasonable explanations for the differences in construction between insect wings and the wings of modern aircraft. The monocoque structure of aircraft wings strongly resists torsion. However, dragonfly

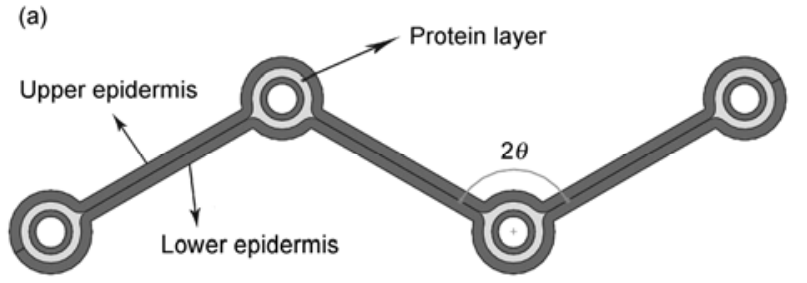

(b)

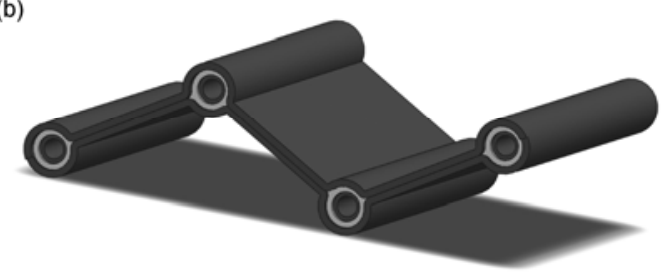

Figure 5 (a) Zigzag cross-section of the wing; (b) 3-D model of the crosssection. Drawn in SolidWorks.

wings must twist between wingbeats to optimize the performance of the aerofoil. The open sandwich vein and the self-adaptable joint or organic junction between the veins and the membrane enable corrugation of the wing structure. This, in turn, allows torsion and results in the camber being set automatically as the wing is twisted. Thus, the organic junction between the vein and membrane contributes to the dragonfly wing's remarkable mechanical behaviors. The microstructure of the organic junction greatly increases warping and torsional rigidity. Further research will focus on quantifying the effect of the joint.

This work was supported by the National Natural Science Foundation of China $(10772091,11072124)$ and the National Basic Research Program of China (2007CB936803, 2010CB631006).

1 Ellington C P. Philos T R Soc B, 1984, 305: 1-15

Newman D J S, Wootton R J. J Exp Biol, 1986, 125: 361-372

Zhao H X, Yin Y J, Zhong Z. Chinese Sci Bull, 2010, 55: 1856-1858

Ennos A R. J Exp Biol, 1988, 140: 137-160

Wang X S, Li Y, Shi Y F. Comp Sci Technol, 2008, 68: 186-192

Song F, Xiao K W, Bai K, et al. Mater Sci Eng A, 2007, 457: 254260

7 Wang X J, Cong Q, Zhang J J, et al. Chinese Sci Bull, 2009, 54: 569_ 575

8 Fang Y, Sun G, Wang T Q, et al. Chinese Sci Bull, 2007, 52: 711716

9 Han Z W, Wu L Y, Qiu Z M, et al. Chinese Sci Bull, 2009, 54: 535540

10 Yang Z X, Dai Z D, Guo C. Chinese Sci Bull, 2010, 55: 771-776

11 Zhao H X, Yin Y J, Zhong Z. Chinese Sci Bull, 2010, 55: 1993-1995

12 Hertel H. Structure, Form, Movement. New York: Reinhold, 1966

13 Sunada S, Zeng L J, Kawachi K. J Theor Biol, 1998, 193: 39-45

14 Rees C J C. Nature, 1975, 256: 200-203

Open Access This article is distributed under the terms of the Creative Commons Attribution License which permits any use, distribution, and reproduction in any medium, provided the original author(s) and source are credited. 